\title{
Avaliação da função fagocitária em remanescentes de esplenectomia subtotal e auto-implante esplênico autógeno

\section{Andy Petroianu}

\section{Introdução}

A esplenectomia priva o organismo de um eficiente filtro fagocitário. Septicemia e outras complicações são conseqüências bem conhecidas da retirada do baço. ${ }^{1-7}$ Com o objetivo de diminuir esses efeitos adversos e preservar as múltiplas funções desse órgão, abordagens conservadoras do baço têm sido cada vez mais
Para diminuir os efeitos adversos da esplenectomia total, abordagens conservadoras devem ser tentadas, quando for possível. Esplen ectomia subtotal e auto-implantes esplêni cos são boas alternativas nessa situação. 0 objetivo do presente estudo foi comparar as fun ções fagocitárias do pólo su perior remanescente de esplenectomia subtotal e de tecido esplênico implantado no omento maior. Esta investigação foi conduzida em 35 ratos Holtzman adultos de ambos os sexos. Todos os animais foram submetidos a esplenectomia subtotal preservando o pólo superior suprido pelos vasos esplenogástricos e a auto-implante do segmento esplên ico removido, no omento maior. A função fagocitária foi verificada por dois métodos diferentes: cintilografia com en xofre coloidal marcado com 99mTc e contagem ao microscópio, de ma crófagos que continham carbono coloidal. Durante os três primeiros meses, a fagocitose foi maior no pólo superior remanescente. Após esse período, não houve diferença entre o pólo superior e o auto-implante. Concluindo, o remanescente de esplenectomia subtotal e o auto-implante esplênico mantêm a função de filtração do baço mediante remoção de partículas coloidais do sangue. Rev. bras. hematol. hemoter. 2003;25(1):25-31.

Palavras-chave: Baço; função fagocitária; macrófago; esplenectomia subtotal; auto-implante esplênico.

Professor Titular do Departamento de Cirurgia da Faculdade de Medicina, UFMG.

Departamento de Cirurgia da Faculdade de Medicina da Universidade Federal de Minas Gerais.

Correspondência para: Prof. Andy Petroianu

Avenida Afonso Pena, 1.626 - apto. 1.901 - 30130-005 - Belo Horizonte- MG

Tel/Fax: (31) 3274-7744 ou 3224-4275 - e-mail: petroian@medicina.ufmg.br 
restante do órgão tornava-se azulado, por isquemia. ${ }^{9}$ Iniciamos nossas pesquisas com trabalhos de anatomia esplênica em cadáveres humanos e, de forma comparativa, em várias espécies animais (camundongos, ratos, cobaias, coelhos, gatos, cães e porcos). ${ }^{17-20}$ Verificamos nesses estudos que o pólo superior do baço apresenta, além do pedículo esplênico, uma outra vascularização, independente do pedículo, constituída pelos vasos esplenogástricos. ${ }^{20}$ Portanto, o pólo superior é a única parte do baço a dispor de dupla vascularização. ${ }^{19}$ Por causa dessa característica, ele mantém a sua vitalidade mesmo quando ocorre obstrução de uma das redes vasculares que o suprem. ${ }^{19-22}$

Após quase cinco anos de estudos experimentais e em cadáver humano sobre a anatomia cirúrgica do baço, sugerimos a esplenectomia subtotal para tratamento da hipertensão porta. ${ }^{10,11}$ Ao longo dos mais de vinte anos em que estamos estudando o baço, estendemos progressivamente a lista de indicações para a esplenectomia subtotal à medida que aumentou a nossa experiência e a literatura forneceu subsídios científicos ao tratamento conservador. Além da hipertensão porta, a esplenectomia subtotal tem sido utilizada para tratamento de trauma esplênico grave, doença de Gaucher, esplenomegalia (metaplasia) mielóide, leucemia linfocítica crônica, afecções corpocaudais do pâncreas e hipodesenvolvimento somático e sexual de origem esplenomegálica. ${ }^{1,2,4,6,7,26-32}$

Quando a esplenectomia é inevitável, podese optar pela preservação de tecido esplênico por meio de auto-implantes. A idéia de semear segmentos de baço em diferentes locais do abdome não é nova. Seus fundamentos estão na esplenose, que é a implantação natural de tecido esplênico oriundo de um trauma em vários locais do organismo. 0 termo esplenose foi introduzido na literatura por Buchbinder e Lipkoff (1939) e, nesse mesmo ano, Calder confirmou o crescimento espontâneo de fragmentos esplênicos na cavidade abdominal. Os primeiros auto-implantes de tecido esplênico no peritônio são atribuídos a Griffini e Tizzioni (1883), que os realizaram em cão. No homem, em cirurgia para tratar trauma esplênico, esse procedimento começou a ser utilizado em 1896, por Albrecht, e depois por Schilling (1907). Atualmente, o grande número de pesquisas conduzidas sobre o implante esplênico autógeno não deixa mais dúvida quanto à sua viabilidade e eficácia funcional. 1,2,4,6,31-41 $\dot{A}$ medida que os cirurgiões se atualizam em cirurgia esplênica, passam a incluir o auto-implante entre as boas alternativas de se manter o tecido esplênico, reduzindo assim as graves adversidades da asplenia cirúrgica. ${ }^{25,41,42}$

0 presente trabalho propõe a avaliação comparativa da função fagocitária do pólo superior remanescente da esplenectomia subtotal e de tecido esplênico auto-implantado no omento maior.

\section{Material e Métodos}

Esta investigação foi conduzida em 35 ratos Holtzman de ambos os sexos. Sua idade variou entre 8 e 10 meses e seu peso médio foi de $378 \pm 43$ gramas. Esses animais foram fornecidos e guardados no Biotério da Faculdade de Medicina da UFMG. Eles permaneceram em gaiolas próprias para ratos, na quantidade de cinco ratos do mesmo sexo por gaiola. Os animais receberam ração para rato e água à vontade. Este trabalho seguiu as recomendações dos Princípios Internacionais de Proteção aos Animais e foi aprovado pela Comissão de Ética do Departamento de Cirurgia da Faculdade de Medicina da UFMG.

Sob anestesia com éter etílico, todos os animais foram submetidos a laparotomia mediana supra e médio-umbilical. 0 baço foi mobilizado para o campo operatório e, após ligadura com fio de seda 4-0 e secção de todos os vasos provenientes do pedículo esplênico, realizou-se a esplenectomia subtotal, preservando-se apenas o pólo superior suprido pelos vasos esplenogástricos. A área cruenta do remanescente esplênico foi hemostasiada com sutura contínua utilizando-se fio de seda 6-0. A parte retirada do baço foi implantada no omento maior com um ponto de fio de seda 4-0. As funções fagocitárias do pólo superior remanescente e do auto-implante foram verificadas por dois métodos diferentes: a cintilografia e a contagem de macrófagos que continham partículas de carbono coloidal. 3,18,43-47 As imagens cintilográficas foram obtidas por 
gama-câmara, uma hora após a injeção de uma solução de enxofre coloidal marcada com $110 \mathrm{MBq}(3 \mathrm{mCi})$ de 99m-tecnécio (99mTc) na veia femoral do rato, sob anestesia com éter etílico.

o carbono coloidal foi obtido de tinta-nanquim, que é constituída por fuligem misturada com goma arábica e perfume. Essa tinta foi colocada em frasco de Becker e secada em estufa a 100 oC. A pasta remanescente foi diluída na proporção de $50 \mathrm{~g} / 100 \mathrm{ml}$ de água. Com o auxílio de uma seringa de um mililitro, injetou-se a solução de carbono coloidal, na quantidade de $1 \mathrm{ml} / \mathrm{kg}$ de peso, na veia femoral do rato, sob anestesia com éter etílico, três horas antes da retirada dos tecidos para estudo.

Os ratos foram mortos com dose excessiva de éter em um tempo que correspondeu a três horas após a injeção do carbono coloidal e uma hora após a injeção do enxofre coloidal marcado com $99 \mathrm{mTC}$. 0 pólo superior esplênico remanescente e o auto-implante foram removidos da cavidade abdominal. Em seguida, foram feitas imagens cintilográficas de ambas as estruturas. ${ }^{48-50}$

Após o estudo cintilográfico, os tecidos esplênicos foram processados para estudo histológico. Os fragmentos esplênicos foram incluídos em parafina. Depois, foram feitos cortes de cinco micra de espessura e processados para fixação em lâmina de microscópio. Utilizaramse duas colorações, hematoxilina e eosina, para estudo morfológico, e verde-luz, para contagem dos macrófagos que continham os pigmentos de carbono coloidal. A contagem dos macrófagos foi feita em 40 campos microscópicos com 0 aumento de 400 vezes. Para cálculo estatístico, considerou-se a média de macrófagos contendo carbono coloidal encontrada nesses campos.

Os ratos foram divididos em sete grupos $(n=5)$, de acordo com o tempo de acompanhamento pós-operatório: um dia, sete dias, trinta dias, sessenta dias, noventa dias, 120 dias e 150 dias. Somente ao fim do período de acompanhamento, os animais receberam os contrastes coloidais para avaliação funcional e foram mortos para remoção do tecido esplênico. As médias dos números de macrófagos que continham carbono coloidal foram comparadas entre os remanescentes esplênicos do pólo superior e do auto-implante estudados no mesmo período pósoperatório. Utilizou-se o teste t de Student com significância para $\mathrm{P}<0,05$.

\section{Resultados}

Todos os animais evoluíram sem anormalidades durante todo o acompanhamento pósoperatório. À laparotomia, foram encontradas aderências em torno do pólo superior esplênico e do auto-implante no omento maior. Essas aderências não impediram a segura remoção de to do o tecido esplênico. Não foram vistas outras anormalidades intra-abdominais.

Todos os exames cintilográficos registraram a presença do contraste coloidal marcado com $99 \mathrm{mTc}$ no pólo superior esplênico. Entretanto, houve intensidade reduzida do nível de radiação durante os dois primeiros meses pós-operatórios. A intensidade da irradiação do pólo superior após noventa dias não foi diferente da observada no fígado. Não foi percebida irradiação emitida pelo auto-implante esplênico até os noventa dias pós-operatórios. Depois de 120 dias, não houve diferença entre a intensidade das imagens do pólo superior e do autoimplante.

A avaliação histológica da morfologia dos auto-implantes foi normal no primeiro dia. Já nos animais acompanhados por sete e trinta dias encontraram-se pouco tecido esplênico e extensas áreas de necrose rodeadas por tecido fibroso. Após sessenta dias, retornou a quase completa predominância de tecido esplênico e, nos grupos que se seguiram aos noventa dias, a arquitetura esplênica foi normal. (Figura 1A) Por outro lado, o pólo superior do baço manteve 0 aspecto histológico normal durante todo o período de acompanhamento pós-operatório.

A tabela 1 apresenta as médias dos números de macrófagos com partículas de carbono coloidal nos pólos superiores esplênicos e nos auto-implantes. Observa-se que os macrófagos esplênicos de todos os remanescentes capturam o colóide desde o primeiro dia pós-operatório. Há, em seguida, uma queda da função fagocitária, que se acentua após trinta dias. Todavia, nos períodos seguintes, há uma recuperação progressiva da capacidade de fagocitose dos 


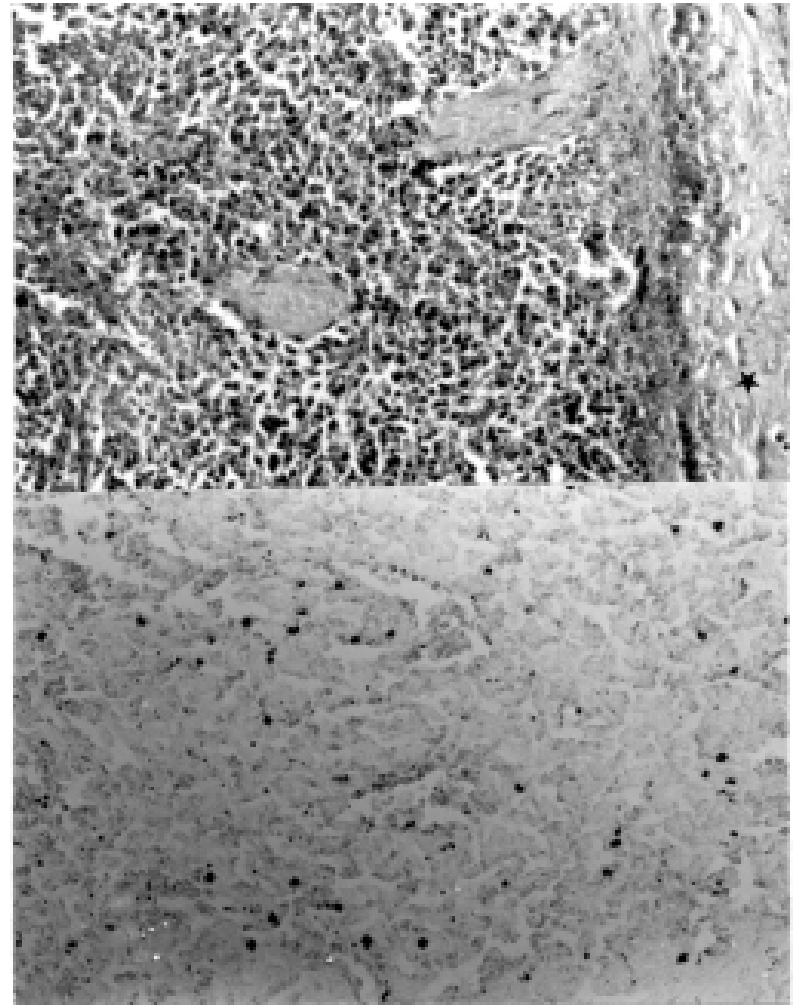

Fig. 1 - Fotomicrografias de cortes de auto-implante esplênico de rato do grupo acompanhado por 120 dias a - Morfologia normal do tecido esplênico. Observa-se a cápsula esplênica(*), com a penetração de uma trave para dentro do parênquima. (HE, $200 \mathrm{X}$ );

b - Parênquima esplênico com destaque para os macrófagos que contêm pigmentos de carbono coloidal em seu interior (pontos pretos espalhados por todo o corte). (Verdeluz, $400 \mathrm{X}$ )

Tabela 1

Número de macrófagos contendo partículas de carbono coloidal. As médias \pm erro padrão da média correspondem à contagem de 40 campos microscópicos com amplificação de $400 \mathrm{X}$

\begin{tabular}{lcc}
$\begin{array}{l}\text { Dia } \\
\text { pós-operatório }\end{array}$ & $\begin{array}{l}\text { Pólo superior } \\
\text { esplênico }\end{array}$ & $\begin{array}{l}\text { Auto - implante } \\
\text { esplênico }\end{array}$ \\
\hline 1 & $226 \pm 43^{*}$ & $52 \pm 19 *$ \\
7 & $96 \pm 36^{*}$ & $18 \pm 13^{*}$ \\
30 & $25 \pm 19 *$ & $4 \pm 4 *$ \\
60 & $264 \pm 58^{*}$ & $39 \pm 33^{*}$ \\
90 & $173 \pm 95$ & $62 \pm 48$ \\
120 & $144 \pm 40$ & $92 \pm 39$ \\
150 & $123 \pm 36$ & $114 \pm 58$
\end{tabular}

*=Diferença significativa para $\mathrm{P}<0,05$ (testet de Student) entre as medias encontradas nos cortes dos polos superiores remanescentes de esplen ectomia subtotal e dos autoimplantes esplênicos no mesmo período de tempo. macrófagos tanto dos pólos superiores esplênicos quanto dos auto-implantes até os 120 dias. (Figura 1B). Depois desse período, a função fagocitária se mantém pouco alterada.

\section{Discussão}

Acredita-se que a depuração sangüínea pelo baço decorra da arquitetura vascular desse órgão. 0 baço remove partículas mediante um mecanismo de captura pelos macrófagos e outras células do sistema mononuclear fagocitário, como os linfócitos. ${ }^{2,29,42}$ De acordo com a literatura, 0 tecido esplênico é capaz de exercer a sua função em qualquer local do organismo em que for implantado. Entretanto, sua totipotencialidade é obtida quando a drenagem se faz para a circulação porta. ${ }^{51}$ Nesse sentido, opta-se por implantar os fragmentos esplênicos no omento maior, no mesentério ou no mesocólon, cujas drenagens sangüíneas se fazem para a veia porta. 1,4,6,7,52-55 Por esse motivo, no presente trabalho, os implantes foram fixados ao omento maior.

Um outro aspecto de grande relevância é a quantidade de tecido esplênico que deve ser implantada. ${ }^{1,56}$ Estudos prévios mostraram a necessidade de pelo menos $25 \%$ do tecido esplênico normal para obter-se a função adequada do baço. ${ }^{29,38,42,54,57,58}$ No caso desta pesquisa, os pólos superiores esplênicos e os auto-implantes continham massa esplênica maior do que $25 \%$ do baço normal de rato. A injeção do carbono coloidal foi realizada duas horas antes da inoculação de enxofre coloidal, portanto três horas antes da morte do animal, em conseqüência de estudo prévio realizado por nós dentro da mesma linha de pesquisa. Ao avaliarmos a influência de um colóide sobre outro colóide na função fagocitária esplênica, percebemos que o enxofre coloidal inibe a fagocitose do carbono coloidal. Por outro lado, a injeção inicial de carbono coloidal não parece interferir na fagocitose do enxofre coloidal. ${ }^{26,53}$

Confirmou-se neste trabalho que os autoimplantes esplênicos não necessitam de anastomose vascular para manter a sua vitalidade. ${ }^{52}$ Após um período de necrose que culmina no primeiro mês pós-operatório, a partir de todos 
os implantes há o desenvolvimento de vasos arteriolares e venosos que se anastomosam naturalmente com os vasos do tecido em que estão implantados. 1,4,6 Quanto ao pólo superior esplênico suprido pelos vasos esplenogástricos, o trauma operatório apresenta pouca influência na função fagocitária desse remanescente esplênico. 2,7,28 É provável que a redução fagocitária observada nos três primeiros meses pósoperatórios possa indicar a queda imunitária verificada no pós-operatório imediato das operações sobre o baço, mesmo quando o procedimento é conservador de tecido esplênico. ${ }^{59} \mathrm{Con}$ tudo, ao se preservar parte do baço, haverá a recuperação funcional esplênica e serão prevenidas as adversidades decorrentes da esplenia.

\section{Agradecimentos}

O autor agradecea o Professor Carlos Jorge Rodrigues Simal pelos exames cintilográficos dos fragmentos esplênicos. Somos gratos também ao Professor Alfredo José Afon so Barbosa, que colocou o laboratório de Anatomia Patológica à nossa disposição para que conduzíssemos a parte histológica do trabalho. Reconhecemos ain da os auxílios fin anceiros do CNPq e da FAPEMIG, fundamentais à realização desta pesquisa.

\section{Abstract}

In order to diminish the adverse effects of total splenectomy, a conservative approach must be performed whenever possible. Subtotal splenectomy and splen ic autotransplantation are good alternatives in such cases. The purpose of the present study was to compare the phagocytic function of the upper pole remnant of subtotal splenectomy and of autologous spleen tissue implanted into the greater omentum. This in vestigation was carried out on 35 adult Holtzman rats of both sexes. All animals were submitted to subtotal splenectomy, preserving the upper pole supplied by the splenogastric vessels and autotransplantation of the removed splenic segment on the greater omentum. The phagocytic function of the splenic remnants was verified by two different methods: scintigraphy with $99 \mathrm{mT}$ c sulfur colloid and microscopic counting of macrophages containing carbon colloid. During the first three months, the phagocytosis was higher in the remnant upper pole.
After this time, there was no difference between the upper poleand the splenic autotransplant In conclusion, the remnant of subtotal splenectomy and the splenic autotran splant maintain the filtering function of the spleen by removing colloid particles from the blood. Rev. bras. hematol. hemoter. 2003; 25(1):25-31.

Key words: Spleen; macrophage; phagocytosis; subtotal splenectomy; autologoussplenic tran splantation.

\section{Referências Bibliográficas}

1. Alvarez FE, Greco RS. Regeneration of the spleen after ectopic implantation and partial splenectomy. Arch Surg 1980;115:772-5.

2. Alvarez $S R$, Escalante $C F$, Ritureto $D C$, Espadas $F L$, Fernandez JM. Assessment of post-splenectomy residual splenic function. Int Surg 1987;72:149-53.

3. Chappell JS. Spleneic preservation. South Afr Med J 1984; 65:12-14.

4. Cooney DR, Dearth JC, Swanson SE, Dewajee MK Relative merits of partial splenectomy, splenic reimmplantation and immunization in preventing postsplenectomy infection. Surgery 1979;86:561-9.

5. Freigenberg Z, Abramovici A, Zer M, Wolloch Y Nathan $H$, Dintsman M. Assessment of splenic funciton in dogs following arterial ligation and autotransplantatioin. Israel J Med Sci 1985;21:579-83.

6. Greco RS, Alvarez FE. Protection against pneumococcal bacteremia by partial splenectomy. Surg Gynecol O bstet 1981;152:67-9.

7. Malangoni MA, Evers BM, Peyton JC. Reticuloendothelia clearance and splenic mononuclear cell populations after resection and autotransplantation. Am 」 Surg 1988;155:298-302.

8. Campos Christo MB. Partial splenectomies in hematologic diseases. Chir Gastroenterol 1993;9 (supp 2):23-9.

9. Petroianu A. Esplenectomia subtotal e anastomose esplenorrenal proximal para o tratamento da hipertensão portal. Rev Bras Cir 1983;73:101-4.

10. Petroianu A. Treatment of portal hypertension by subtotal splenectomy and central splenorenal shunt. Postgrad Med J 1988;64:38-41.

11. Petroianu A. Subtotal splenectomy and portal-varicea disconnection in the treatment of portal hypertension. Canad J Surg 1993;36:251-4.

12. Petroianu A. Esplenectomia subtotal preservando o pólo superior suprido pelos vasos esplenogástricos. Rev Col Bras Cir 1994:21:21-6.

13. Petroianu A. Treatment of cystadenoma of the pancreatic tail by distal pancreatectomy and subtotal splenectomy. Dig Surg 1995;12:259-61. 
14. Petroianu A. Subtotal splenectomy in Gaucher's disease. Eur J Surg 1996;162:511-3.

15. Petroianu A. Subtotal splenectomy for treatment of patients with myelofibrosis and myeloid metaplasia. Int Surg 1996:81:177-9.

16. Resende V, Petroianu A. Estudo funcional tardio do auto-implante esplênico após trauma complexo do baço humano. Rev Col Bras Cir 2001;28:165-70.

17. Petroianu A. Avaliação imaginológica da esplenectomia subtotal e anastomose esplenorrenal centralizada para o tratamento da hipertensão portal esquistossomática. Rev Med Minas Gerais 1993;3:106-8.

18. Petroianu A, Ferreira VLM, Barbosa AJA. Morphology and viability of the spleen after subtotal splenectomy. Braz J Med Biol Res 1989;22:491-5.

19. Petroianu A. Splenic viability after segmental devascularization. Rev Paul Med 1992;110:39-41.

20. Petroianu A, Petroianu S. Anatomy of splenogastric vessels in patients with schistosomal portal hypertension. Clin Anat 1994;7:80-3.

21. Petroianu A, Antunes LJ. Immune profiles in hepatosplenic schistosomiasis mansoni after surgical treatments. J Int Med Res 1998;26:43-9.

22. Petroianu A, Petroianu, LPG. A twelve-year-follow-up of subtotal splenctomy and central splenorenal shunt for treatment of schistosomatic portal hypertension. Folha Méd 1998;116:107-9.

23. Petroianu A, Silva RG, Simal CJR. Late postoperative follow-up of patients submitted to subtotal splenectomy. Am Surg 1997;63:735-40.

24. Petroianu A, Berindoague Neto R. Laparoscopic subtotal splenectomy Surg Laparosc Endosc Percut Tech 2002; no prelo.

25. Petroianu A. Subtotal splenectomy for the treatment of retarded growth and sexual development assciated with splenomegaly. Minerva Chir 2002; no prelo.

26. Corazza GR, Tarozzi C, Vaira D, Frisoni M, Gasbarrini $G$. Return of splenic function after splenectomy. Br Med J 1984;289:861-6

27. Drew PA, Kiroff GK, Ferrante A. Alterations in immunoglobulin synthesis by peripheral blood mononuclear cells from splenectomized patients with and without splenic regrowth. J Immunol 1984;132:191-6.

28. Nielsen JL, Sakso $\mathrm{P}$, Sorensen $\mathrm{FH}, \mathrm{H}$ ansen $\mathrm{HH}$. Demonstration of splenic functions following splenectomy and autologous spleen implantatioon. Acta Chir Scand 1984;150:469-73.

29. Patel JM, Williams JS, Naim JO. The effect of site and technique of splenic tissue reimplantation on pneumococcal clearance from the blood. J Pediatr Surg 1986;21:877-80

30. Pearson HA, Johnston D, Smith KA, Touloukian RJ. Return os splenic function after splenectomy for trauma. N Eng J Med 1978;298:1.389-92.

31. Petroianu A, Vidigal FM, Costa VC. Splenic auto- transplantation in Gaucher's disease. Dig Surg 2000; 17:181-3.

32. Petroianu A, Petroianu LPG. Splenic autotransplantation combined with splenectomy and portal-variceal disconnection in the treatment of portal hypertension. Am Surg 2002; no prelo.

33. Haklar G, Demirel M, Peker 0 . The functional assessment of autotransplanted splenic tissue by its capacity to remove oxidatively modified erythrocytes. Clin Chim Acta 1997:258:201-8.

34. Hathaway JM, Harley RA, Self. Immunological function in post-traumatic splenosis. Clin Immunol Immuno pathol 1995;74:143-50.

35. Leemans R, Beekhuis $\mathrm{H}$, Timens W. Fc-receptor function after human splenic autotransplantation. Br J Surg 1996; 83:543-6.

36. Leemans R, Harms G, Rijkers GT. Spleen autotransplantation provides restoration of functional splenic lymphoid compartments and improves the humoral immune response to pneumococcal polysaccharide vaccine. Clin Exp Immunol 1999;117:596-604.

37. Leemans R, Manson W, Snijder JAM. Immune response capacity after human splenic autotransplantation. Ann Surg 1999;229:279-85.

38. Marques RG, Petroianu A, Oliveira MBN, Bernardo MF Portela MC, Boasquevisque EM. Distribuição de Escherichia coli nos órgãos dos sistema mononuclear fagocitário após esplenectomia total e auto-implante esplênico em rato. Rev Col Bras Cir 2002; no prelo.

39. Timens W, Leemans R. Splenic autotransplantation and the immune system. Ann Surg 1992;215:256-60.

40. Silva RG, Petroianu A. Influência das operações sobre o baço na distribuição da Escherichia coli no sistema mononuclear fagocitário. Rev Col Bras Cir 2002; no prelo.

41. Petroianu A, Berindoague Neto R. Laparoscopic splenic autotransplantation $\mathrm{Br}$ J Surg 2002; no prelo.

42. Drew PA, Clayers MTR, Jamieson GG. The value of splenic autotransplantation. Arch Surg 1990;125:1.224.

43. Petroianu A, Barbosa AJA. Quantitative studies on the macrophage phagocytosis in whole spleen and in remnant of subtotal splenectomy. Med Sci Res 1991;19:373-5.

44. Petroianu A, Simal CJR, Barbosa AJA. Splenic macrophage phagocytic function after subtotal splenectomy in dog. Med Sci Res 1992;20:127-8.

45. Petroianu A, Simal CJR, Barbosa AJA. Impairment of phagocytosis by mammalian splenic macrophages by 99mTc sulphur colloid. Med Sci Res 1992;20:847-9.

46. Petroianu A, Simal, CJR. Shifts of the reticuloendothelia system uptake pattern induced by carbon colloid. Med Sci Res 1993;311-2.

47. Petroianu A, Barbosa AJA. Splenic preservation based on preserved histology and phagocytic function using upper short gastric vessels. Am J Surg 1995;170:702. 
48. Armas R, Thakur ML, Gottschalk A. A simple method of spleen imaging with $99 \mathrm{mTc}$-labeled erythrocytes. Radiology 1979;132:215-6.

49. Dunn EK, Vaquer RA, Strashun AM. Attempts at clarification of splenic visualization on reticuloendothelial images. Clin Nucl Med 1989;14:647-50.

50. Hermann HJ, Winkel K. Scintigraphy of the spleen. Lymphology 1977;10:115-9.

51. Livingston $C D$, Levine $B A$, Sirinek, KR. Site of splenic autotransplantation affects protection from sepsis. Am J Surg 1983;146:734-7.

52. Clayer MTR, Drew P, Leong A. The vascular supply of splenic autotransplants. J Surg Res 1992;53:475-84.

53. Clayer MTR, Drew P, Leong A. IgG-mediated phagocytosis in regenerated splenic tissue. Clin Exp Immunol 1994;97:242-7.

54. linuma $\mathrm{H}$, Okinaga $\mathrm{K}$, Sato $\mathrm{S}$, et al. Optimal site and amount of splenic tissue for autotransplantation. J Surg Res 1992;53:109-16.
55. Weber T, Hanisch E, Baum. Late results of heterotopic autotransplantation of splenic tissue into the greate omentum. World J Surg 1998;22:883-9.

56. Yiong G, Han G, Xian-Tao K, Immunoglobulins and complement in splenectomized and autotransplanted subjects. Ann Med 1989:21:265-7

57. Witte $\mathrm{CL}$, Witte $\mathrm{MH}$. Enlargement of splenic implants. Surgery 1995;117:357.

58. Zoli G, Corazza GR, D'Amato G. Splenic autotransplantation after splenectomy. Br J Surg 1994;81 716-8.

59. Shokouh-Amiri MH, Kaharazami A, Saber, SR. Phagocyte function after splenic autotransplantation. Arch Surg 1990;125:595-7.

Recebido: 25/07/02

Aceito: $06 / 12 / 02$ 